\title{
Letter to the editor - Pathology of childhood: From newborn to adolescence
}

\author{
Vlad DIMA ${ }^{1 *}$, Anca A. SIMIONESCU ${ }^{2,3}$ \\ ${ }^{1}$ Department of Neonatology, Filantropia Clinical Hospital, Bucharest, Romania \\ 2 "Carol Davila" University of Medicine and Pharmacy, Bucharest, Romania \\ ${ }^{3}$ Department of Obstetrics and Gynecology, Filantropia Clinical Hospital, Bucharest, Romania \\ *Coordinator of the supplement
}

Neonatal-perinatal medicine is a complex specialty that cannot be reduced to the simple transposition of pediatric practice in a neonatal setting. Close collaboration between obstetricians, neonatologists, pediatrician, pediatric surgeon and other specialists are essential for solving complex cases. We propose this special issue of the Romanian Journal of Practical Medicine because we want to provide an overview of the current research on practical care improvement pursuit, especially by multidisciplinary collaboration.

In fact, in practice, the neonatologists and pediatricians may face the following situations:

- normal newborn cases, where the role of the neonatologist is essential in initiating and establishing early mother-infant interaction and attachment, avoiding mother anxiety and in assuring the family that the neonate is not sick and in counseling for breastfeeding

- infants requiring neonatal intensive care units (NICU) admission, anesthesia and surgical intervention, palliative care, or which will have unpredictable evolution and long-term hospitalization. Extremely preterm infants or infants with severe neonatal pathologies need aggressive intensive care and are at high risk of morbidity and mortality.

- children presenting to an emergency pediatric department or children need special considerations and monitoring in pediatric department.

In 2020, Romania has reported 163,320 livebirth making a decline from years before [1]. Romania has one of the highest infants mortality rates in the EU [1], a sign that there is still much to be done at this level. In the last decade, neonatal medicine has made great strides by introducing complex treatment methods. The collaboration with the obstetricians, specialists in maternal-fetal medicine and geneticists determined the integration of this specialty in the multidisciplinary management.

As a level III maternity in Bucharest, Filantropia Clinical Hospital has reported in 2020 almost 5,000 livebirths, with an average of 3 days of hospitalization. More than 100 newborns were born prematurely and 10 cases of newborns with prenatal diagnosed abnormalities.

Care of the extremely preterm birth babies $(<27$ weeks of pregnancy) is one of the most challenging aspects of perinatal medicine, both for clinicians, families and hospital staff. Many advances have been made since modern neonatal paediatrics started in the 1960s with the introduction of mechanical assisted ventilation [2]. However, perinatal care at extremely preterm gestations will always need to be individualised and should be led by senior staff obstetrics and neonatology.

Most neonates admitted to the NICU are premature babies, have perinatal asphyxia, intrauterine growth restriction (less than 5 percentile), or have a health condition that needs special care in collaboration with other specialists.

Neonatologists can face neonatal consequences of maternal diseases: feto-maternal infections, infants born from mothers with diabetes mellitus, fetal anemia, and maternal allo-immunisation. In addition, Streptococcus B infection, cytomegalovirus, congenital rubella, syphilis or herpes require special investigations and appropriate care [3].

Acute intoxication and systemic autoimmune disorders (lupus, rheumatoid arthritis, psoriasis, myasthenia gravis etc.) have a higher prevalence in women, particularly during their childbearing age. If maternal disease is characterized by the presence of IgG auto-antibodies, these can cross the placenta with possible antibody-mediated consequences to the fetus, fetal abnormalities, thrombocytopenia, leucopenia, hemolytic anemia or other [4]. 
All the pathologies encountered during neonatal period should be considered for follow up by pediatricians during the childhood. Pediatricians will also have an essential role when it comes to follow up for the preterm babies. One should not forget that the challenges of prematurity are not over at the moment of the discharge from maternity. Follow-up will continue with the help of the pediatricians, ophthalmologists and neurologists during the first years of life. There are numerous studies that showed a strong link between prematurity and neurologic or psychiatric disorders for example, even during the adolescence.

Pediatricians are facing constant challenges when it comes to obesity and other pathologies linked to infancy behaviors. Obesity has increased in the last decades.
Obese children are more likely to develop preventable non-communicable diseases like diabetes and cardiovascular diseases at a younger age [5].

So we can not talk about neonatology without taking into account the later role of pediatrics during the life of each baby.

In conclusion, pregnancy, childbirth, and the perinatal period are considered high-risk periods for the health of mothers and their newborns. Therefore, the quality of maternal-fetal and newborn care may contribute to reduce maternal and perinatal mortality and morbidity through rapid management and stabilization and early diagnosis and management of pregnancy-related complications.

Conflict of interest: none declared Financial support: none declared

\section{REFERENCES}

1. Eurostat data, accessed on 15 July 2021. Available at: http://ec.europa.eu.

2. Swyer PR, Reiman RC, Wright JJ. Ventilation and ventilatory mechanics in the newborn: methods and results in 15 resting infants. J Pediatr. 1960 May;56:612-22.

3. Simionescu AA, Daia-lliescu S. Pregnancy, systemic lupus erythematosus and a short communication on labor complications as new onset of the disease. Ro J Rheumatol. 2021;30(2):61-67.

4. Simionescu AA, Ana Maria Alexandra Stanescu AMA. Group B streptococcal infections during pregnancy and perinatal consequences: what do we need to determine more than a bacteriological analysis? Ro J Infect Dis. 2021;24(2):71-75.
5. Sahoo K, Sahoo B, Choudhury AK et al. Childhood obesity: causes and consequences. J Family Med Prim Care. 2015;4(2):187-92. 